\title{
Multidomain Feature Fusion for Varying Speed Bearing Diagnosis Using Broad Learning System
}

\author{
Tingting Wu, ${ }^{1}$ Yufen Zhuang, ${ }^{1}$ Bi Fan $\left(\mathbb{D},{ }^{1}\right.$ Hainan Guo, ${ }^{1}$ Wei Fan $\left(\mathbb{D},{ }^{2}\right.$ Cai Yi, ${ }^{3}$ \\ and Kangkang $\mathrm{Xu}^{4}$ \\ ${ }^{1}$ College of Management, Shenzhen University, Shenzhen 518061, China \\ ${ }^{2}$ School of Mechanical Engineering, Jiangsu University, Zhenjiang 212013, China \\ ${ }^{3}$ State Key Laboratory of Traction Power, Southwest Jiaotong University, Chengdu 610031, China \\ ${ }^{4}$ School of Electromechanical Engineering, Guangdong University of Technology, Guangzhou 510006, China
}

Correspondence should be addressed to Bi Fan; fanbi@outlook.com

Received 18 December 2020; Revised 30 December 2020; Accepted 8 January 2021; Published 29 January 2021

Academic Editor: Tangbin Xia

Copyright ( $\odot 2021$ Tingting Wu et al. This is an open access article distributed under the Creative Commons Attribution License, which permits unrestricted use, distribution, and reproduction in any medium, provided the original work is properly cited.

Bearing is one of the most critical mechanical components in rotating machinery. To identify the running status of bearing effectively, a variety of possible fault vibration signals are recorded under multiple speeds. However, the acquired vibration signals have different characteristics under different speeds and environment interference, which may lead to different diagnosis results. In order to improve the fault diagnosis reliability, a multidomain feature fusion for varying speed bearing diagnosis using broad learning system is proposed. First, a multidomain feature fusion is adopted to realize the unified form of vibration characteristics at different speeds. Time-domain and frequency-domain features are extracted from the different speeds vibration signals. Then, the broad learning system is employed with the fused features for classification. Our experimental results suggest that, compared with other machine learning models, the proposed broad learning system model, which makes full use of the fused feature, can improve the diagnosis performance significantly in terms of both accuracy and robustness analysis.

\section{Introduction}

Rolling elements bearings are important machine elements that are widely used in railway wheels, wind turbines, gearboxes, pumps, and helicopter transmissions [1]. The running state of axle bearings, as the core component of high-speed trains, plays an important role in the safe and stable operation of the high-speed rail [2]. The railway axle bearings can support rotating machine elements and transfer loads of machine components under the fast-running state of a train. As the speed increases, the rolling bearings are operated under harsh conditions like heavy loads, long-term alternating stresses, and natural wear [3]. The axle bearings operating under these circumstances are prone to failures such as pitting, spalls, and axle burn-off [4]. If these bearing faults are ignored, this may lead to equipment damage and even cause serious safety accidents [5]. Thus, it is necessary to detect the bearing fault and give early warning before affecting daily operations. Effective fault diagnosis of bearings can prevent potential accidents and reduce unexpected economic loss [4]. It has been found that bearing faults are often accompanied by abnormal vibration signals, such as outer ring inner surface faults, cage faults, roller faults, and the compound faults [6]. Therefore, analyzing and mining diagnostic information from vibration data have important scientific significance and high application value.

At present, it is impossible to identify the faults from the vibration signal directly. The vibration signal is vulnerable to the operating environment and unknown factors, such as the track impact, speed change, and the vehicle body vibration [7]. It is necessary to eliminate the interference and extract useful information that can reflect the health of the bearing. Feature extraction is a key step to solve this problem. It can transform the original vibration signals onto the statistical parameters reflecting diverse symptoms of bearing defects [8]. Many methods have been developed, such as Fourier 
transform (FT), short-time Fourier transform (STFT), wavelet transform (WT), and wavelet packet decomposition (WPD) [9-15]. Bouzida et al. [16] employed discrete wavelet transform to obtain information about the health state of a system from stator signal over a wide range of frequencies. $\mathrm{Li}$ et al. [17] proposed a feature extraction and evaluation method based on statistical features. Zhang et al. [18] proposed an ant colony algorithm for synchronous feature selection in intelligent fault diagnosis of rotating machinery. Cong et al. [19] used the slip matrix construction method to extract features. However, the characteristics of vibration signal of bearing under different rotating speeds are rarely discussed. Some researchers have developed several fault diagnosis methods according to different speeds, respectively $[2,20]$. This method seems to work well dealing with different speeds, but it lacks convenience in practice. In addition, the characteristics of the vibration signal across different speeds have not been studied.

The vibration signal can be regarded as an expression of a moving process in time domain and frequency domain [3]. Operating speed of the bearings will also impact the moving process [21]. Therefore, the inherent characteristic of vibration signal of variable speed should be extracted from the time and frequency domain together to achieve better fault diagnosis performance [22]. Thus, a unified description of multidomain fusion for varying speed should be studied [21]. The accuracy of detection mainly depends on the quality of features extracted from the vibration data. Therefore, a multidomain features fusion is proposed to present the inherent characteristics of the vibration signal for varying speed comprehensively. The common time and frequency features [20] involve mean value, variance, maximum, root mean square, etc. They can capture intrinsic information about bearing defects. For example, mean value in time domain represents central trend, and the variance in frequency domain conveys message of signal changes. We chose STFT to extract time domain features for its compatible implementation in the fast Fourier transform. Meanwhile, WPD was one of the most widely used and advanced technologies to analyze signal data in frequency domain. These two methods were combined to extract time and frequency features.

In recent years, machine learning algorithms, such as broad learning system (BLS), artificial neural network (ANN), extreme learning machine (ELM), support vector machine (SVM), and logistic regression (LR) have been applied successfully in fault diagnosis of roller bearings [23-26]. Sobie et al. [21] proposed a novel application of dynamic time warping (DTW) to bearing fault classification. Toma et al. [27] used $\mathrm{KNN}$, decision tree, and random forest to evaluate the bearing faults. Zhang et al. [28] proposed a novel hybrid model using permutation entropy (PE), ensemble empirical mode decomposition (EEMD), and support vector machine (SVM) to detect roller bearing faults. Recently, BLS has received a lot of attention due to its outstanding performance in fault diagnosis. Zhao et al. [2] employed BLS as classifier to detect the bearing faults. Zhao et al. [29] proposed semisupervised broad learning system for fault diagnosis. However, these studies either directly use BLS without considering the inherent characteristics of the data or only consider the frequency- domain characteristics of the signal. It seems that the present work cannot make full use of BLS in fault diagnosis.

In this paper, we propose a multidomain feature fusion for varying speed bearing diagnosis using BLS. The diagnostic power of the method is attributable to two features: First, we extract the intrinsic vibration characteristics at multiple speeds. A multidomain fusion is adopted to realize the unified form of vibration characteristics; second, the kernel-based broad learning system has short computing time and good generalization ability. Different from previous studies, the original vibration data at different speeds are converted into unified time-domain and frequencydomain data. As far as we know, few studies have adopted this method to extract diagnostic information from different speeds at one time. Obviously, with multidomain fusion, the vibration data of different speeds can be explored thoroughly under a unified framework to obtain more dynamic fault information. The experiment results illustrate that the proposed method is significantly superior to some other machine learning models. Meanwhile, the diagnostic power of BLS with varying speed data is stronger than that with one speed, which may provide solid evidence that varying speed data are of great significance to fault diagnosis.

The remainder of this paper is organized as follows. Section 2 introduces the framework of the proposed diagnosis method. The proposed multidomain feature fusion and BLS are provided in Section 2. The empirical study is given in Section 3. Finally, Section 4 offers concluding work and implications for further research.

\section{Methodology}

2.1. Analytical Framework. The general scheme of applying the multidomain feature fusion for varying speed bearing diagnosis is shown in Figure 1. The proposed method includes three major steps:

(1) Obtain the Vibration Signals under Different Rotating Speeds. The raw data were collected from the sensors and segmented into training dataset and testing dataset.

(2) Multidomain Feature Fusion. The acquired vibration data were preprocessed with time-domain and frequency-domain transformation. The time statistical characteristics and the frequency spectra of the vibration signals provide potentially valuable fault information.

(3) Fault Diagnosis Using BLS. The obtained fused features were utilized to train the BLS and other machine learning classification models. Then the testing dataset is used to validate their performance.

2.2. Multidomain Feature Fusion for Varying Speed. Feature extraction plays an important role in fault diagnosis. In a condition monitoring system, the bearing is often running under different speeds. These vibration signals of varying speed contain a variety of possible fault information. In particular, the dynamic characteristics of different speeds 


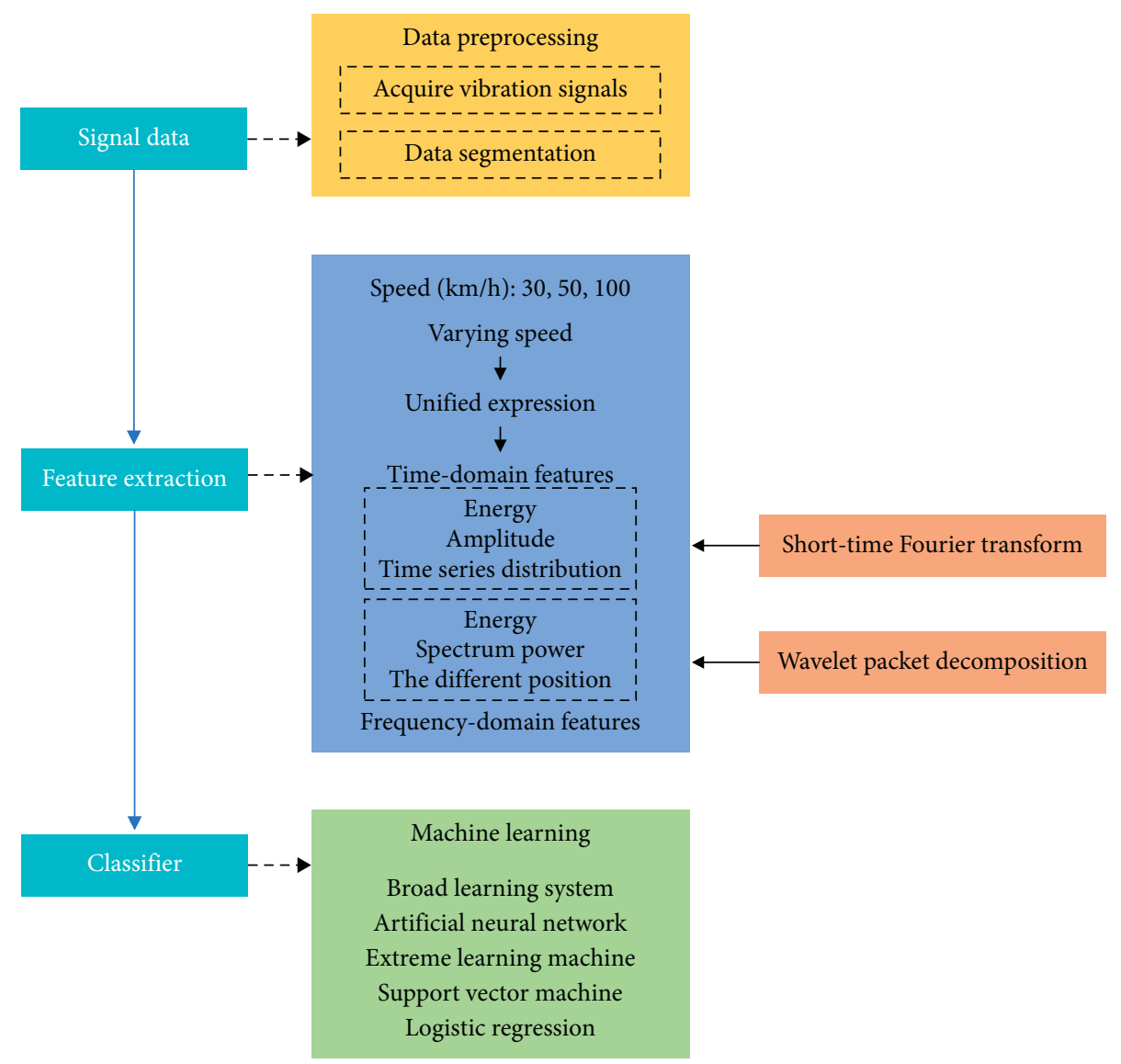

FIgURE 1: The framework of the proposed method.

should be considered together. The purpose of multidomain fusion is to produce more reliable and more accurate information from varying speed.

In order to capture the diagnostic information, a total of 50 features are extracted from each speed vibration signal, 18 time-domain features, and 32 frequency-domain features as listed in Tables 1 and 2, respectively. Table 1 presents the description of 18 time-domain features, including mean value, mean square, peak-to-peak value, impulse factor, and crest factor. Table 2 presents the definition of 32 frequencydomain features, including frequency center and RMS frequency. Among them, $p_{1}$ and $p_{16}-p_{18}$ denote the amplitude and energy of time-domain features. $p_{7}$ and $p_{11}-p_{12}$ reflect the time series distribution. $p_{19}$ is the energy of frequency-domain features. $p_{21}-p_{24}, p_{26}$, and $p_{30}-p_{33}$ are the spectrum power. $p_{25}$ and $p_{27}-p_{29}$ show the different positions of the main frequencies. The remaining features are extracted by wavelet packet analysis and expressed as equations $p_{34}-p_{50}$.

2.3. Broad Learning System. Broad learning system (BLS) was proposed by Professor Chen [30]. It is an incremental learning algorithm based on the random vector function link neural network (RVFLNN). For this method, firstly, the original data are projected in the feature space using a linear function and transformed into features notes of the BLS. Then enhancement notes are generated by feature notes randomly through a nonlinear activation function. Next, all feature nodes and enhancement nodes are directly connected to the output coefficients, which can be obtained by the ridge regression. Finally, the structure of BLS is constructed after the output weight is obtained. Compared with other deep networks, the structure of BLS is simple. The BLS establishes a flat network structure consisting of feature nodes, enhancement nodes, and output coefficient matrix. It can quickly extract features from new data and reduce retraining time. Therefore, the flat network of BLS can be more effective and efficient in classification and regression problems without deep architecture [29]. The BLS structure is shown in Figure 2 [30].

(1) For the input data $X$, the linear transformation function mapping is used to project data, which become the $i$ th mapped features $Z_{i}$.

$$
Z_{i}=\varnothing_{i}\left(X W_{e i}+\beta_{e i}\right)
$$

where $W_{e i}$ and $\beta_{e i}$ are the random weights with the proper dimensions. Denote $Z_{i} \equiv\left[Z_{1}, \ldots, Z_{n}\right]$, which is the concatenation of all the first $i$ groups of mapping features and $n$ is the number of groups of feature nodes; $i=1, \ldots, n$. 
TABle 1: Time-domain features.

\begin{tabular}{lcc}
\hline$p_{1}=(1 / N) \sum_{i=1}^{N} x_{i}$ & $p_{2}=\max \left(x_{i}\right)$ & $p_{3}=\min \left(x_{i}\right)$ \\
$p_{4}=(1 / N) \sum_{i=1}^{N}\left(x_{i}-p_{1}\right)^{2}$ & $p_{5}=\sqrt{p_{4}}$ & $p_{6}=\left((1 / N) \sum_{i=1}^{N} x_{i}^{2}\right)^{1 / 2}$ \\
$p_{7}=p_{6} /(1 / N) \sum_{i=1}^{N}\left|x_{i}\right|$ & $p_{8}=(1 / N) \sum_{i=1}^{N}\left(x_{i}-p_{1} / p_{5}\right)^{4}$ & $p_{9}=(1 / N) \sum_{i=1}^{N}\left(x_{i}-p_{1} / p_{5}\right)^{3}$ \\
$p_{10}=p_{2}-p_{3}$ & $p_{11}=\max \left(\left|x_{i}\right|\right) / p_{6}$ & $p_{12}=\max \left(\left|x_{i}\right|\right) /(1 / N) \sum_{i=1}^{N}\left|x_{i}\right|$ \\
$p_{13}=p_{2} / p_{7}$ & $p_{14}=p_{2} / p_{6}$ & $p_{15}=p_{8} / p_{6}^{4}$ \\
$p_{16}=(1 / N) \sum_{i=1}^{N}\left|x_{i}\right|$ & $p_{17}=\max \left(\left|x_{i}\right|\right)$ & $p_{18}=\left((1 / N) \sum_{i=1}^{N} \sqrt{\left|x_{i}\right|}\right)^{2}$ \\
\hline
\end{tabular}

Note: $x_{i}$ denotes a signal data for $i=1,2, \ldots, N . N$ indicates the number of signal points.

TABLE 2: Frequency-domain features.

\begin{tabular}{lcc}
\hline$p_{19}=(1 / K) \sum_{i=1}^{K} s_{i}$ & $p_{20}=\left((1 / K) \sum_{i=1}^{K} s_{i}^{2}\right)^{1 / 2}$ & $p_{21}=(1 / K-1) \sum_{i=1}^{K}\left(s_{i}-p_{19}\right)^{2}$ \\
$p_{22}=\sqrt{p_{21}}$ & $p_{23}=\sum_{i=1}^{K}\left(s_{i}-p_{19}\right)^{3} / K\left(p_{22}\right)^{3}$ & $p_{24}=\sum_{i=1}^{K}\left(s_{i}-p_{19}\right)^{4} / K\left(p_{22}\right)^{2}$ \\
$p_{25}=\sum_{i=1}^{K} f_{i} s_{i} / \sum_{i=1}^{K} s_{i}$ & $p_{26}=\left(\sum_{i=1}^{K}\left(f_{i}-p_{25}\right)^{2} s_{i} / K\right)^{1 / 2}$ & $p_{27}=\left(\sum_{i=1}^{K} f_{i}^{2} s_{i} / \sum_{i=1}^{K} s_{i}\right)^{1 / 2}$ \\
$p_{28}=\left(\sum_{i=1}^{K} f_{i}^{4} s_{i} / \sum_{i=1}^{K} f_{i}^{2} s_{i}\right)^{1 / 2}$ & $p_{29}=\sum_{i=1}^{K} f_{i}^{2} s_{i} /\left(\sum_{i=1}^{K} s_{i} \sum_{i=1}^{K} f_{i}^{4} s_{i}\right)^{1 / 2}$ & $p_{30}=p_{26} / p_{25}$ \\
$p_{31}=\sum_{i=1}^{K}\left(f_{i}-p_{25}\right)^{3} s_{i} / K\left(p_{26}\right)^{3}$ & $p_{32}=\sum_{i=1}^{K}\left(f_{i}-p_{25}\right)^{4} s_{i} / K\left(p_{26}\right)^{4}$ & $p_{33}=\sum_{i=1}^{K}\left(f_{i}-p_{15}\right)^{1 / 2} s_{i} / K\left(p_{26}\right)^{1 / 2}$ \\
$p_{34}-p_{49}=E_{i} / \sum_{i=1}^{16} \sum E_{i}$ & $p_{50}=-\sum_{i=1}^{16} E_{i} \log _{2} E_{i}$ & \\
\hline
\end{tabular}

Note: $s_{i}$ shows a spectrum for $i=1,2, \ldots, K . K$ describes the number of spectrum lines. $f_{i}$ is the frequency value of the $k$ th spectrum line.

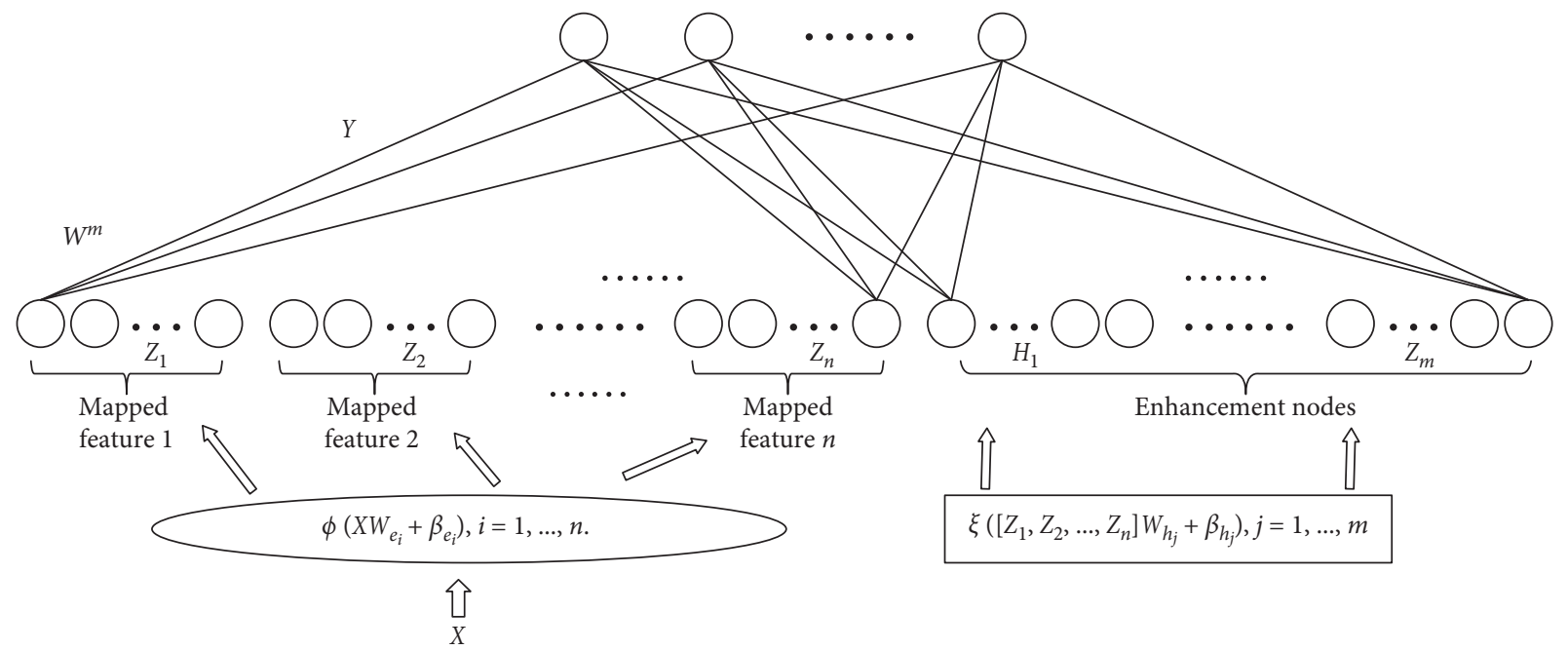

Figure 2: The BLS structure.

(2) Similarly, the $j$ th group of enhancement nodes $H_{j}$ can be generated using the nonlinear function transformation

$$
H_{j}=\xi_{i}\left(Z_{n} W_{h j}+\beta_{h j}\right),
$$

where $W_{h j}$ and $\beta_{h j}$ are random weight coefficients with appropriate dimensions. The concatenation of all the first $j$ groups of enhancement nodes is denoted as $H^{m} \equiv\left[H_{1}, \ldots, H_{m}\right] . \quad m$ is the total number of enhancement nodes; $j=1, \ldots, m$.

Hence, the BLS can be represented as the equation of the form

$$
\begin{aligned}
Y & =\left[Z_{1}, \ldots, Z_{n} \mid \xi\left(Z_{n} W_{h_{1}}+\beta_{h_{1}}\right), \ldots, \xi\left(Z_{n} W_{h_{m}}+\beta_{h_{m}}\right)\right] W^{m} \\
& =\left[Z_{1}, \ldots, Z_{n} \mid H_{1}, \ldots, H_{m}\right] W^{m} \\
& =\left[Z^{n} \mid H^{m}\right] W^{m},
\end{aligned}
$$

where $W^{m}=\left[Z^{n} \mid H^{m}\right]^{+} Y . W^{m}$ are the connecting weights coefficients and can be easily computed using the ridge regression.

\section{Experiment and Analysis}

3.1. Experiment Data and Environment. In order to verify the effectiveness of the proposed method, the experiments were carried out on a rotary machine experimental platform, as shown in Figure 3. The platform can simulate various operating conditions of trains with infinite long rail through double-wheel reverse scrolling. The test rig can simulate various operating conditions of trains. The fault states of train can be imitated through artificially seeded defects in bench experiments. Experiments were designed with three faulty bearings and one normal bearing. Under each type of fault defects, the bearing was running at the speeds of $30 \mathrm{~km} /$ $\mathrm{h}, 50 \mathrm{~km} / \mathrm{h}$, and $100 \mathrm{~km} / \mathrm{h}$ for 2 minutes, respectively, and the 10 seconds' vibration data were collected with the sampling frequency of $10 \mathrm{kHz}$. For each bearing defect in certain speed, 10,000 pieces of data were sampled. The fault types and speeds are shown in Table 3. According to the sampling frequency and rotating speed, the number of sampling points included in a cycle during the bearing rotation is calculated. Therefore, the original data were 


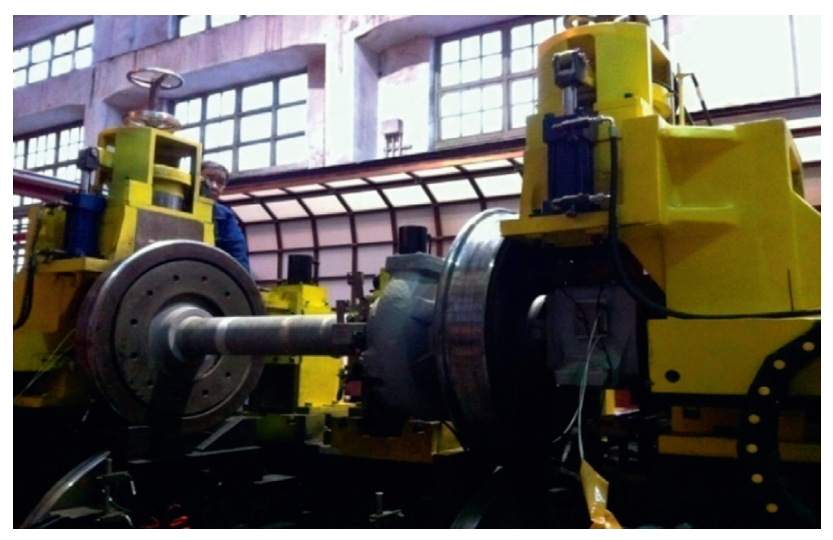

FIgURE 3: Experimental setup.

TABle 3: Description of bearing dataset.

\begin{tabular}{lccc}
\hline Name & Fault type & Speed $(\mathrm{km} / \mathrm{h})$ & Fault classification \\
\hline$Z_{1} C_{1}$ & Outer race defects & $30,50,100$ & 1 \\
$Z_{2}$ & Cage defect & $30,50,100$ & 2 \\
$Z_{2}$ & Pin roller defect & $30,50,100$ & 3 \\
$Z_{3}$ & Normal & $30,50,100$ & 4 \\
\hline
\end{tabular}

TABLe 4: Comparison of classification accuracy.

\begin{tabular}{lccccc}
\hline Models & $\mathrm{ZC}_{1}$ & $\mathrm{ZC}_{2}$ & $\mathrm{ZC}_{3}$ & $\mathrm{ZC}_{4}$ & Average accuracy (\%) \\
\hline BLS & 96.23 & 97.60 & 94.49 & 98.74 & 96.2 \\
ANN & 87.28 & 83.17 & 86.59 & 89.68 & 86.68 \\
ELM & 73.47 & 92.15 & 84.21 & 88.92 & 84.69 \\
SVM & 92.99 & 94.34 & 97.97 & 88.86 & 93.54 \\
LR & 74.13 & 88.65 & 82.82 & 89.32 & 83.73 \\
\hline
\end{tabular}

divided into smaller datasets according to different faults and speeds.

In order to capture the dynamic diagnostic information, there are 18 time-domain features and 32 frequency-domain features extracted from each vibration signal, as listed in Tables 1 and 2. These features are fused to form the multidomain features. The obtained fused features can be utilized to train the BLS for fault diagnosis.

3.2. Fault Classification. To further verify the effectiveness of the proposed method, the multidomain fusion features are used to train the BLS, ANN, SVM, ELM, and LR as the classification model. The SVM classifier is a margin-based supervised machine learning method. SVM model can be effectively applied in nonlinearly separable data. The radial basis function was used in SVM model. ELM is a single hidden layer neural network algorithm and has been widely used in many fields because of simple mathematical description, lower computational burden, and faster learning speed [31]. ANN was inspired by biological nervous systems function of the man brain. It is suitable for complex natural systems by establishing relationships among highly anomalous nonlinear variables and producing sophisticated, accurate, and reliable results [32]. In this study, the number of hidden neurons of ELM and ANN models was determined by trial-and-error testing. LR is capable of bearing fault diagnosis for its high accuracy in the nearly linearly separable data.

The accuracy of each fault is used to evaluate the performance of these classifiers. The fault diagnosis performances of these models are shown in Table 4 . The comparison results of accuracy of each fault and average accuracy are provided. The proposed BLS model has the highest average accuracy in testing compared to other machine learning methods. Furthermore, BLS constantly outperforms other methods for each fault, followed by SVM, ANN, and ELM, whereas LR ranks last.

The robustness of the proposed method is also assessed. Due to the random variables in BLS model, we ran the experiment ten times to obtain the average performance of BLS and analyzed its robustness according to standard deviation of accuracy. The results are provided in Figure 4 . The standard deviations of accuracy are as follows: $0.009\left(\mathrm{ZC}_{1}\right), 0.007\left(\mathrm{ZC}_{2}\right)$, $0.012\left(\mathrm{ZC}_{3}\right), 0.005\left(\mathrm{ZC}_{4}\right)$, and 0.002 (Average). Generally, the performance of the BLS is stable, since the standard deviations from the accuracy of each fault are small. The standard deviation from average accuracy is smaller than others.

To further examine the effectiveness of the multidomain fusion for varying speed, the training data are classified into three types: " $30 \mathrm{~km} / \mathrm{h}$," " $50 \mathrm{~km} / \mathrm{h}$," and " $100 \mathrm{~km} / \mathrm{h}$." These are vibration data under different speeds. When one dataset is used to train the BLS, the other two datasets are tested. For 


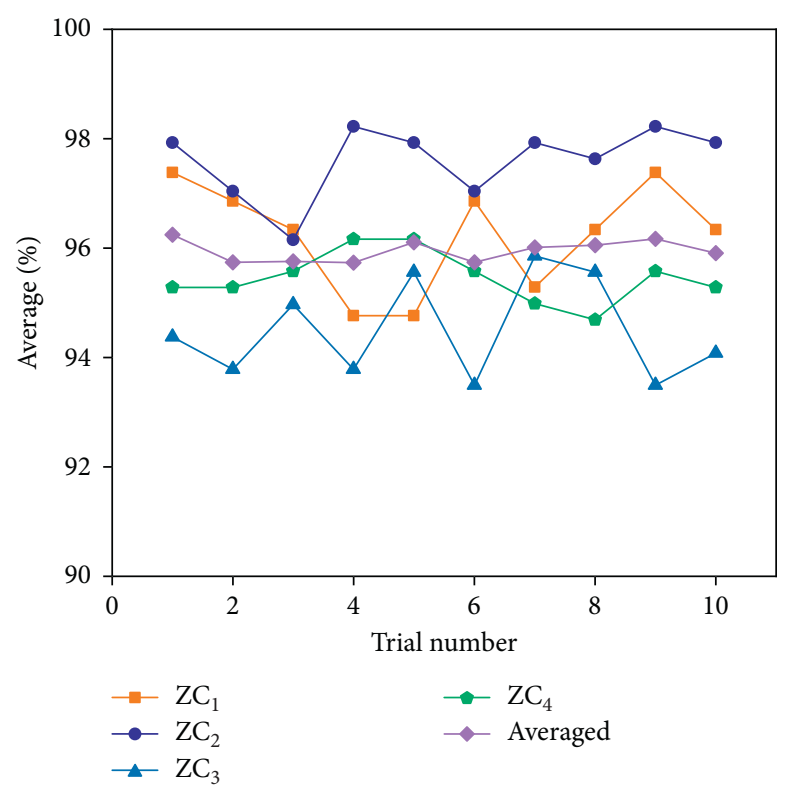

FIgURE 4: Results of 10 trials using BLS.

TABLE 5: Classification comparison of different combinations.

\begin{tabular}{|c|c|c|c|}
\hline Models & Training dataset $(\mathrm{km} / \mathrm{h})$ & Testing dataset $(\mathrm{km} / \mathrm{h})$ & Average accuracy (\%) \\
\hline \multirow{6}{*}{ BLS } & 30 & 50 & 94.12 \\
\hline & 30 & 100 & 79.24 \\
\hline & 50 & 30 & 98.03 \\
\hline & 50 & 100 & 85.63 \\
\hline & 100 & 30 & 74.94 \\
\hline & 100 & 50 & 91.32 \\
\hline \multirow{6}{*}{ ANN } & 30 & 50 & 87.33 \\
\hline & 30 & 100 & 72.44 \\
\hline & 50 & 30 & 80.02 \\
\hline & 50 & 100 & 71.16 \\
\hline & 100 & 30 & 79.46 \\
\hline & 100 & 50 & 90.68 \\
\hline \multirow{6}{*}{ ELM } & 30 & 50 & 82.64 \\
\hline & 30 & 100 & 68.65 \\
\hline & 50 & 30 & 95.25 \\
\hline & 50 & 100 & 81.46 \\
\hline & 100 & 30 & 78.12 \\
\hline & 100 & 50 & 70.18 \\
\hline \multirow{6}{*}{ SVM } & 30 & 50 & 78.91 \\
\hline & 30 & 100 & 80.14 \\
\hline & 50 & 30 & 94.33 \\
\hline & 50 & 100 & 94.42 \\
\hline & 100 & 30 & 80.95 \\
\hline & 100 & 50 & 91.70 \\
\hline \multirow{6}{*}{ LR } & 30 & 50 & 83.05 \\
\hline & 30 & 100 & 64.28 \\
\hline & 50 & 30 & 95.51 \\
\hline & 50 & 100 & 87.98 \\
\hline & 100 & 30 & 63.35 \\
\hline & 100 & 50 & 83.37 \\
\hline
\end{tabular}

comparison with the BLS, we conducted other experiments using SVM, ANN, ELM, and LR to test the effectiveness of different combinations. The experimental results are recorded in Table 5. Generally, the performance of the five models with different combinations is not good. BLS is more accurate, followed by SVM, ANN, LR, and ELM. When the 
speed of training dataset is higher than that of the testing dataset, the accuracy of the model seems better. For example, when the training dataset is " $50 \mathrm{~km} / \mathrm{h}$ " and testing dataset is " $30 \mathrm{~km} / \mathrm{h}$," the classification accuracy of five models is good. In general, we can get some evidence that (1) there are declines in diagnosis accuracy; (2) the training and testing data with a large speed difference make the diagnosis more difficult; (3) different speeds contain different dynamic characteristics for fault diagnosis. It has been revealed that the classifier developed on one speed data may not be capable of good diagnosis on other speed data On the contrary, the method proposed in this paper has good performance of fault diagnosis under multispeed with one classifier.

\section{Conclusion}

In this paper, we proposed a diagnosis framework using multidomain feature fusion and machine learning to detect faults from vibration data at different speeds. The study considers the dynamic characteristics of multiple speeds together to obtain more comprehensive fault diagnosis information. Features extracted from time and frequency domains are combined into one vector to present the characteristics of multiple speeds. Then BLS is developed on these fused features for fault diagnosis. The experimental results suggest that the proposed BLS models with multidomain features can significantly improve the forecasting performance compared with other machine learning methods. Beside the accuracy, the robustness of the proposed BLS is also analyzed. Moreover, it is also verified that the classifier developed on one speed data may not be able to perform well on other speed data. However, this study does have some limitations, mainly because we only solved this problem at a certain level. There are still some problems to be investigated. The relationship between the vibration data of different speeds is still unknown. How to develop a diagnostic model for all speeds is also a problem Furthermore, the model could deal with multiple domain data such as multiple sensors and speeds. These questions should be studied in the future.

\section{Data Availability}

Data are available upon request to the corresponding author by e-mail.

\section{Conflicts of Interest}

The authors declare that they have no conflicts of interest.

\section{References}

[1] R. B. Randall, Vibration-Based Condition Monitoring: Industrial, Aerospace and Automotive Applications, John Wiley \& Sons, Hoboken, NJ, USA, 2011.

[2] H. Zhao, J. Zheng, J. Xu, and W. Deng, "Fault diagnosis method based on principal component analysis and broad learning system," IEEE Access, vol. 7, pp. 99263-99272, 2019.

[3] C. Zheng, D. Wang, W. Fan, K.-L. Tsui, and J. Lin, "EEMDbased steady-state indexes and their applications to condition monitoring and fault diagnosis of railway axle bearings," Sensors, vol. 18, no. 3, p. 704, 2018.

[4] C. Yi, J. Lin, W. Zhang, and J. Ding, "Faults diagnostics of railway axle bearings based on IMF's confidence index algorithm for ensemble EMD," Sensors, vol. 15, no. 5, pp. 10991-11011, 2015.

[5] A. Heng, A. C. C. Tan, J. Mathew, N. Montgomery, D. Banjevic, and A. K. S. Jardine, "Intelligent condition-based prediction of machinery reliability," Mechanical Systems and Signal Processing, vol. 23, no. 5, pp. 1600-1614, 2009.

[6] S. Tan, H. A. Toliyat, and X. Li, "Condition monitoring and fault diagnosis of electrical motors-a review," IEEE Transactions on Energy Conversion, vol. 20, no. 4, pp. 719-729, 2005.

[7] J. R. Stack, T. G. Habetler, and R. G. Harley, "Effects of machine speed on the development and detection of rolling element bearing faults," IEEE Power Electronics Letters, vol. 1, no. 1, pp. 19-21, 2003.

[8] M. Kang, J. Kim, J.-M. Kim, A. C. C. Tan, E. Y. Kim, and B.-K. Choi, "Reliable fault diagnosis for low-speed bearings using individually trained support vector machines with kernel discriminative feature analysis," IEEE Transactions on Power Electronics, vol. 30, no. 5, pp. 2786-2797, 2015.

[9] Y. Li, H. Zhao, W. Fan, and C. Shen, "Extended noise resistant correlation method for period estimation of pseudo-periodic signals," IEEE Transactions on Instrumentation and Measurement, pp. 1-1, 2021.

[10] W. Fan, Q. Zhou, J. Li, and Z. Zhu, “A wavelet-based statistical approach for monitoring and diagnosis of compound faults with application to rolling bearings," IEEE Transactions on Automation Science and Engineering, vol. 15, no. 4, pp. 1563-1572, 2017.

[11] Y. Fan, L. Li, L. Chen, and Z. Xu, "Bearing Fault Detection Via B-spline Constructed Sparse Method," IEEE Transactions on Instrumentation and Measurement, pp. 1-1, 2021.

[12] G. Chen, Q.-Y. Li, D.-Q. Li, Z.-Y. Wu, and Y. Liu, "Main frequency band of blast vibration signal based on wavelet packet transform," Applied Mathematical Modelling, vol. 74, pp. 569-585, 2019.

[13] D. Li, "Theory of communication. Part 1: the analysis of information," Journal of the Institution of Electrical EngineersPart III: Radio and Communication Engineering, vol. 93, no. 26, pp. 429-441, 1946.

[14] H. Gao, L. Liang, X. Chen, and G. Xu, "Feature extraction and recognition for rolling element bearing fault utilizing shorttime Fourier transform and non-negative matrix factorization," Chinese Journal of Mechanical Engineering, vol. 28, no. 1, pp. 96-105, 2015.

[15] Y. Liang, G. Xu, L. Liang, and K. Jiang, "Detection of weak transient signals based on wavelet packet transform and manifold learning for rolling element bearing fault diagnosis," Mechanical Systems and Signal Processing, vol. 54-55, pp. 259-276, 2015.

[16] A. Bouzida, O. Touhami, R. Ibtiouen, A. Belouchrani, M. Fadel, and A. Rezzoug, "Fault diagnosis in industrial induction machines through discrete wavelet transform," IEEE Transactions on Industrial Electronics, vol. 58, no. 9, pp. 4385-4395, 2010.

[17] W. Li, Z. Zhu, F. Jiang, G. Zhou, and G. Chen, "Fault diagnosis of rotating machinery with a novel statistical feature extraction and evaluation method," Mechanical Systems and Signal Processing, vol. 50-51, pp. 414-426, 2015.

[18] X. Zhu, W. Chen, B. Wang, and X. Chen, "Intelligent fault diagnosis of rotating machinery using support vector machine with ant colony algorithm for synchronous feature selection 
and parameter optimization," Neurocomputing, vol. 167, pp. 260-279, 2015.

[19] F. Chen, W. Zhong, S. Tong, N. Tang, and J. Chen, "Research of singular value decomposition based on slip matrix for rolling bearing fault diagnosis," Journal of Sound and Vibration, vol. 344, pp. 447-463, 2015.

[20] Z. Chow and W. Li, "Multisensor feature fusion for bearing fault diagnosis using sparse autoencoder and deep belief network," IEEE Transactions on Instrumentation and Measurement, vol. 99, pp. 1-10, 2017.

[21] C. Zhong, C. Freitas, and M. Nicolai, "Simulation-driven machine learning: bearing fault classification," Mechanical Systems and Signal Processing, vol. 99, pp. 403-419, 2018.

[22] B. Li, M.-Y. Chow, Y. Tipsuwan, and J. C. Hung, "Neuralnetwork-based motor rolling bearing fault diagnosis," IEEE Transactions on Industrial Electronics, vol. 47, no. 5, pp. 1060-1069, 2000.

[23] J. B. Ali, "Application of empirical mode decomposition and artificial neural network for automatic bearing fault diagnosis based on vibration signals," Applied Acoustics, vol. 89, no. 3, pp. 16-27, 2015.

[24] Y. Tian, J. Ma, C. Lu, and Z. Wang, "Rolling bearing fault diagnosis under variable conditions using LMD-SVD and extreme learning machine," Mechanism and Machine Theory, vol. 90, pp. 175-186, 2015.

[25] K. Ma, X. Song, and D. Xue, “A roller bearing fault diagnosis method based on hierarchical entropy and support vector machine with particle swarm optimization algorithm," Measurement, vol. 47, pp. 669-675, 2014.

[26] D. H. Pandya, S. H. Upadhyay, and S. P. Harsha, "Fault diagnosis of rolling element bearing by using multinomial logistic regression and wavelet packet transform," Soft Computing, vol. 18, no. 2, pp. 255-266, 2014.

[27] R. N. Toma, A. E. Prosvirin, and J.-M. Kim, "bearing fault diagnosis of induction motors using a genetic algorithm and machine learning classifiers," Sensors, vol. 20, no. 7, p. 1884, 2020.

[28] X. Xhang, Y. Liang, and J. Zhou, "A novel bearing fault diagnosis model integrated permutation entropy, ensemble empirical mode decomposition and optimized SVM," Measurement, vol. 69, pp. 164-179, 2015.

[29] H. Zhao, J. Zheng, W. Deng, and Y. Song, "Semi-supervised broad learning system based on manifold regularization and broad network," IEEE Transactions on Circuits and Systems I: Regular Papers, vol. 67, no. 3, pp. 983-994, 2020.

[30] C. L. P. Chen and Z. Liu, "Broad learning system: an effective and efficient incremental learning system without the need for deep architecture," IEEE Transactions on Neural Networks \& Learning Systems, vol. 99, pp. 1-15, 2017.

[31] K.-K. Xu, H.-D. Yang, and C.-J. Zhu, "A novel extreme learning machine-based Hammerstein-Wiener model for complex nonlinear industrial processes," Neurocomputing, vol. 358, pp. 246-254, 2019.

[32] J. K. Ali, "Neural networks: a new tool for the petroleum industry?," in Proceedings of the European Petroleum Computer Conference, Aberdeen, UK, March 1994. 